\title{
Specificity as a Graph-Based Estimator of Cross-Entropy and KL Divergence
}

\author{
Carole Twining and Chris J. Taylor \\ ISBE, University of Manchester, Manchester, UK. \\ carole.twining@manchester.ac.uk
}

\begin{abstract}
There are various methods for building statistical models of shape or shape and appearance from training data. Quantitative comparison of such methods requires a method of evaluating the quality of the fit between the model pdf and the training data. One quantitative graph-based measure which has been used for this purpose is the specificity, and the associated measure of generalisation. In this paper we consider the large-numbers limit of the specificity, and derive expressions which show that specificity can be considered as a graph-based estimator of cross-entropy. We also give explicit expressions for the various constants involved. We perform simple experiments using artificial data, and show that these limiting relations hold good even for relatively small numbers of training examples. We hence establish a proper theoretical context for the previously ad hoc concept of specificity.
\end{abstract}

\section{Introduction}

In statistical modelling of either shapes or images, the input training set of shapes can be considered as a set of points lying in some (Euclidean) shape or image space. A statistical model constructed from this training set is then a pdf defined on this space. If we are working with automatic model-building methods, different methods applied to the same training data can produce slightly different models. If we wish to compare different model-building strategies, we hence require some quantitative measure that enables us to compare the training set of points, and the model pdf. Note that we cannot use directly methods that depend on estimating the pdf of the training set, since this is in effect the very model-building stage that we wish to evaluate.

One such measure is the specificity [2], as defined below. The construction is that some large set of sample points is generated from the model pdf, distributed according to that pdf. For each member of the sample set, the distance to the nearest member of the training set is computed. The idea being that for a good model of the training data, the instances generated by the model should be specific, that is, they should be close to those seen in the training set. Note that we cannot use statistically-defined distances (e.g., the Mahalanobis distance) to measure degree of closeness, since they will be small by construction. The advantage of this construction is that it can in principle be applied to any distribution of points and any generative statistical model. The global minimum specificity of any point set wrt the model hence corresponds to a $\delta$-function distribution at points. However, we are not interested in this global minimum, but rather the constrained problem of minimising the specificity between the data and a fitted model, wrt variations in the data, such as those caused by varying the correspondence between shapes from a single training set. 
The specificity has been shown to be useful in practice. However, despite this success, what was lacking was a sound theoretical basis for its usage. For instance, it would be advantageous if it were possible to compare directly specificities measured for different sizes of training set. Such a theoretical basis is derived in this paper. It is shown that by taking the limit of a large number of training examples, specificity can be viewed as a graph-based estimator of the cross-entropy between the pdf that generated the training set, and the pdf of the model. This hence relates specificity to the already-existing body of work on graph-based estimators of entropies and divergences (e.g., [4, 3, 5]).

\section{The Large-Numbers Limit of Specificity}

\subsection{Derivation}

We start with the usual definition of the specificity of a point set and a statistical or probabilistic model as defined by other authors (e.g., [2]). Consider a set of $n$ training points lying in $\mathbb{R}^{d}: X^{(n)}=\left\{x_{a} \varepsilon \mathbb{R}^{d}: a=1, \ldots n\right\}$. We also have a model of this data, a pdf $f(z)$, from which we generate a large sample set of points $Y^{(M)}=\left\{y_{\mu}: \mu=1, \ldots M\right\}$, distributed according to the model pdf. The specificity is then defined as:

$$
\hat{S}_{1}\left(X^{(n)} ; Y^{(M)}\right)=\frac{1}{M} \sum_{\mu=1}^{M} \min _{a}\left(\left\|y_{\mu}-x_{a}\right\|\right),
$$

where $\|\cdot\|$ is just the Euclidean distance in $\mathbb{R}^{d}$. The generalisation measure is defined similarly, but with the rôles of training and sample points reversed.

An obvious generalisation is to take the sum of powers of the distances, rather than just the sum, which gives what we will call the $\lambda$-specificity:

$$
\hat{S}_{\lambda}\left(X^{(n)} ; Y^{(M)}\right)=\frac{1}{M} \sum_{\mu=1}^{M} \min _{a}\left(\left\|y_{\mu}-x_{a}\right\|\right)^{\lambda} .
$$

Mathematically, we see that this is just a Monte Carlo estimator of the integral $\lambda$-specificity:

This integral can then be decomposed thus:

$$
\lim _{M \rightarrow \infty} \hat{S}_{\lambda}\left(X^{(n)} ; Y^{(M)}\right)=S_{\lambda}\left(X^{(n)} ; f\right)=\int_{\mathbb{R}^{d}} f(z) \min _{a}\left(\left\|z-x_{a}\right\|\right)^{\lambda} d z .
$$

$$
S_{\lambda}\left(X^{(n)} ; f\right)=\int_{\mathbb{R}^{d}} f(z) \min _{a}\left(\left\|z-x_{a}\right\|\right)^{\lambda} d z=\sum_{a=1}^{n} \int_{\Omega_{a}\left(X^{(n)}\right)} f(z)\left(\left\|z-x_{a}\right\|\right)^{\lambda} d z,
$$

where $\Omega_{a}\left(X^{(n)}\right)=\left\{z:\left\|z-x_{a}\right\| \leq\left\|z-x_{b}\right\| \forall x_{b} \varepsilon X^{(n)}\right\}$ is the Voronoi cell belonging to the training point $x_{a}$, generated by the set $X^{(n)}$.

To proceed further, we must suppose that the training set $X^{(n)}$ has actually been generated by some process with pdf $g(z)$. Let us consider some infinitesimal volume $\Delta z$ about a point $x_{a}$, and let $n \rightarrow \infty$. Then, by definition, the number of points of $X^{(n)}$ within this volume tends to $n g\left(x_{a}\right) \Delta z$, and the average point-spacing within this volume tends to $l_{a}=\left(n g\left(x_{a}\right)\right)^{-\frac{1}{d}}[1]$. Furthermore, locally about $x_{a}$, the distribution of training points becomes indistinguishable in the limit from those generated by an infinite uniform Poisson point process ${ }^{2}$. We will denote the relevant uniform Poisson process by $\mathcal{P}_{a} \doteq \mathcal{P}\left(x_{a}, n g\left(x_{a}\right)\right)$, which is a process of intensity $n g\left(x_{a}\right)$ about the point $x_{a}$. If $n$ is large enough, we can also neglect the variation of $f(z)$ across the Voronoi cell $\Omega_{a}$, to obtain:

\footnotetext{
${ }^{1}$ Note that we here suppose that the intrinsic dimensionality of $X^{(n)}$ is the same as $d$

${ }^{2}$ This is just the objective method approach of Steele [7], where the limiting behaviour of local functionals on finite point sets is described in terms of related functionals defined on infinite Poisson processes.
} 


$$
\sum_{a=1}^{n} \int_{\Omega_{a}\left(X^{(n)}\right)} f(z)\left(\left\|z-x_{a}\right\|\right)^{\lambda} d z \underset{n \rightarrow \infty}{\longrightarrow} \sum_{a=1}^{n} f\left(x_{a}\right) \int_{\Omega_{a}\left(\mathscr{P}_{a}\right)}\left(\left\|z-x_{a}\right\|\right)^{\lambda} d z,
$$

where $\Omega_{a}\left(\mathcal{P}_{a}\right)$ is now the Voronoi cell of $x_{a}$ for the process $\mathcal{P}_{a}$. This integral across the Voronoi cell is replaced by its expectation value:

$$
\mathbb{E}\left[\int_{\Omega_{a}\left(P_{a}\right)}\left(\left\|z-x_{a}\right\|\right)^{\lambda} d z\right]=\left(l_{a}\right)^{\lambda+d} \mathbb{E}\left[\int_{\Omega_{0}\left(\mathcal{P}_{1}\right)}(\|z\|)^{\lambda} d z\right]
$$

where we have used simple scaling to replace the Poisson process $\mathcal{P}_{a}$ of intensity $n g\left(x_{a}\right)$ by a Poisson process of unit intensity $\mathcal{P}_{1}$, and translational invariance to measure distances from the origin rather than from $x_{a}$. We define:

$$
\beta_{d, \lambda} \doteq \mathbb{E}\left[\int_{\Omega_{0}\left(\mathcal{P}_{1}\right)}(\|z\|)^{\lambda} d z\right],
$$

and hence obtain the required estimate of the limit:

$$
\begin{array}{ll} 
& \lim _{n \rightarrow \infty} S_{\lambda}\left(X^{(n)} ; f\right)=\beta_{d, \lambda} \sum_{a=1}^{n} f\left(x_{a}\right)\left(l_{a}\right)^{\lambda+d}, \text { where: } l_{a}=\left(n g\left(x_{a}\right)\right)^{-\frac{1}{d}} \\
& =\frac{\beta_{d, \lambda}}{n^{\frac{\lambda}{d}}} \sum_{a=1}^{n} f\left(x_{a}\right) g^{-\frac{\lambda}{d}}\left(x_{a}\right)\left(l_{a}\right)^{d} \underset{n \rightarrow \infty}{\longrightarrow} \frac{\beta_{d, \lambda}}{n^{\frac{\lambda}{d}}} \int_{\mathbb{R}^{d}} f(z) g^{-\frac{\lambda}{d}}(z) d z . \\
\text { That is: } \quad & \lim _{n \rightarrow \infty}\left[n^{\frac{\lambda}{d}} S_{\lambda}\left(X^{(n)} ; f\right)\right]=\beta_{d, \lambda} \int_{\mathbb{R}^{d}} f(z) g^{-\frac{\lambda}{d}}(z) d z .
\end{array}
$$

In order to relate this to other quantities, we define the divergences ${ }^{3}$ :

$$
\begin{array}{r}
D_{\lambda}(f, g) \doteq \frac{d}{\lambda} \log \int_{\mathbb{R}^{d}} f(z) g^{-\frac{\lambda}{d}}(z) d z, \quad \widetilde{D}_{\lambda}(f, g) \doteq D_{\lambda}(f, g)-D_{\lambda}(f, f), \\
\lim _{\lambda \rightarrow 0} D_{\lambda}(f, g)=-\int_{\mathbb{R}^{d}} f(z) \log g(z) d z=H(f, g), \lim _{\lambda \rightarrow 0} \widetilde{D}_{\lambda}(f, g)=D_{K L}(f, g),
\end{array}
$$

where $H(f, g)$ is the cross-entropy and $D_{K L}(f, g)$ the Kullback-Leibler (KL) divergence. We hence obtain an estimator for cross-entropy using the Specificity ${ }^{4}$ :

$$
H(f, g)=\lim _{\lambda \rightarrow 0} D_{\lambda}(f, g) \approx \lim _{\lambda \rightarrow 0}\left(\frac{d}{\lambda}\left[\log \left(n^{\frac{\lambda}{d}} S_{\lambda}\left(X^{(n)} ; f\right)\right)-\log \beta_{d, \lambda}\right]\right) .
$$

However, we can only use this estimator if we know the value of $\beta_{d, \lambda}$ (7).

\subsection{The Meaning of $\beta_{d, \lambda}$}

The important fact about the constant $\beta_{d, \lambda}$ is that it does not depend on the pdfs $f(z)$ and $g(z)$. This is because of the limiting behaviour noted above, which enables $\beta_{d, \lambda}$ to be defined purely in terms of uniform Poisson point processes.

There is another link we can establish. Consider the definition (7) again:

$$
\beta_{d, \lambda} \doteq \mathbb{E}\left[\int_{\Omega_{0}\left(P_{1}\right)}(\|z\|)^{\lambda} d z\right]
$$

\footnotetext{
${ }^{3}$ The term divergence relates to the separation of two distributions. It cannot be called a metric or a distance since in general divergences are not symmetric, that is, $D(f, g) \neq D(g, f)$.

${ }^{4}$ The corresponding limit of the generalisation measure is then just the other cross-entropy $H(g, f)$.
} 
We can view this integral as the limit of a sampling process, where the point $z$ is generated randomly and uniformly. But this means that generating $z$ is just adding another point to the uniform Poisson process $\mathcal{P}_{1}$. Hence $\beta_{d, \lambda}$ should be equal to the expectation value of the nearest-neighbour distance for such a process. And it is relatively simple to calculate this value explicitly; in [8], Wade calculates the large-n limit for the k-nearest-neighbour power- $\lambda$ graph length for a point process with a non-uniform distribution, and the term (in our notation) that is the generalised equivalent of our $\beta_{d, \lambda}$ is given by:

$$
C(d, \lambda, k)=\left(v_{d}\right)^{-\frac{\lambda}{d}} \frac{d}{d+\lambda} \frac{\Gamma\left(1+k+\frac{\lambda}{d}\right)}{\Gamma(k)} \text {, where: } v_{d}=2 \pi^{\frac{d}{2}} / d \Gamma\left(\frac{d}{2}\right),
$$

$v_{d}$ being the volume of the unit ball in $d$ dimensions. We have considered just the firstnearest-neighbour here, so would expect that $\beta_{d, \lambda}=C(d, \lambda, 1)$. As a check on this deduction, we note that $\beta_{d, \lambda}$ can be calculated explicitly from equation (7), using the methods of Brakke [1] (see Appendix), and that the value obtained is precisely that given above.

There is a further link we can establish, between cross-entropy and entropy. If we take the distributions $f(z)$ and $g(z)$ to be the same, the cross-entropy just reduces to the Shannon entropy of $f(z)$. If we also consider our original definition of the specificity (1), we see that this then becomes an estimate of the length of the $\lambda$-power nearest-neighbour graph for $n+1$ points, divided by the number of points. Albeit with a rather strange sampling scheme, in that the $n$ points in $X^{(n)}$ are held fixed, whilst the $(n+1)^{t h}$, the point drawn from $Y$, is generated $M$ times, and only the distance from this $(n+1)^{t h}$ point is measured. We hence obtain the previously-known result of the nearest-neighbour graph length on a set of points as an estimator of Shannon entropy [4] as a special case.

Finally, we note that the divergence $D_{\lambda}(f, g)$ (9) we defined earlier is of a slightly different form to the Rényi $\alpha$-divergences $R_{\alpha}(f, g)$ [6]:

$$
D_{\lambda}(f, g)=\frac{d}{\lambda} \log \int_{\mathbb{R}^{d}} f(z) g^{-\frac{\lambda}{d}}(z) d z, \quad R_{\alpha}(f, g)=\frac{1}{\alpha-1} \log \int_{\mathbb{R}^{d}} f^{\alpha}(z) g^{1-\alpha}(z) d z .
$$

Hero et al. [3, 5] estimated $R_{\alpha}(f, g)$ using graph-based methods. Note however that they were interested in the divergence of two point sets, rather than the divergence of a point set and a pdf. Both tend towards the same expression in the limit $(\gamma \rightarrow 0$ or $\alpha \rightarrow 1)$.

\subsection{Generalised Specificity \\ 2.3.1 Other Neighbourhoods}

The above analysis considered just the case of first nearest-neighbours. It is clear however that the notion of specificity can be further generalised, to either the $j^{\text {th }}$ nearestneighbour (NN) or $k$-nearest-neighbours (NNs) cases. For example, for the case of the $j^{\text {th }}$-NN specificity, equation (2) becomes:

$$
\hat{S}_{\lambda}^{j^{\prime}}\left(X^{(n)} ; Y^{(M)}\right)=\frac{1}{M} \sum_{\mu=1}^{M}\left(d_{j}\left(y_{\mu}, X^{(n)}\right)\right)^{\lambda}
$$

where $d_{j}\left(y_{\mu}, X^{(n)}\right)$ is just the Euclidean distance between $y_{\mu}$ and its $j^{t h}$ nearest-neighbour in $X^{(n)}$. The $k$-NNs specificity is then given by:

$$
\hat{S}_{\lambda}^{k}\left(X^{(n)} ; Y^{(M)}\right)=\sum_{j=1}^{k} \hat{S}_{\lambda}^{j^{\prime}}\left(X^{(n)} ; Y^{(M)}\right) .
$$

It can be seen that the analysis of $\S 2.1$ follows through as before, except that the way that $\mathbb{R}^{d}$ is partitioned is slightly more complicated. The final result is that given previously, except that the constant $\beta_{d, \lambda}$ is replaced by the relevant constants as given by Wade ([8], page 6, Theorem 2.1) for the case of $j^{t h}-\mathrm{NN}$ and $k$-NNs graphs. 


\subsubsection{Other Distances}

Equation (1) can be generalised in another way. Suppose rather than Euclidean distance we have a translation invariant distance-like quantity $\mathcal{D}(x, y)=\mathcal{D}(x-y)$ which varies monotonically with Euclidean distance. This of course includes the powers of Euclidean distance used previously. The nearest-neighbour specificity wrt $\mathcal{D}$ is then:

$$
\hat{S}^{\mathcal{D}, 1}\left(X^{(n)} ; Y^{(M)}\right)=\frac{1}{M} \sum_{\mu=1}^{M} \min _{a} \mathcal{D}\left(y_{\mu}, x_{a}\right) .
$$

The analysis then follows as previously. Note that because of the properties of $\mathcal{D}(\cdot)$ defined above, the Voronoi cells of $\mathcal{D}(\cdot)$ correspond exactly with the Euclidean Voronoi cells. Equation (6) is replaced by:

$$
\mathbb{E}\left[\int_{\Omega_{a}\left(P_{a}\right)} \mathcal{D}\left(z, x_{a}\right) d z\right] .
$$

The translation invariance allows us to rewrite this in terms of Voronoi cells around the origin. We need to know how the distance scales before we can reduce it to a cell of a uniform Poisson process $\Omega_{\mathbf{0}}\left(\mathcal{P}_{1}\right)$.

Let us take as a non-trivial example the logarithm of the Euclidean distance: $\mathcal{D}(z)=\log z$. Then the specificity becomes:

$$
\begin{aligned}
& \lim _{n \rightarrow \infty} \hat{S}_{\log }^{1}\left(X^{(n)} ; Y^{(M)}\right)=-\frac{1}{d} \log n-\frac{1}{d} \int_{\mathbb{R}^{d}} f(z) \log g(z) d z+\mathbb{E}\left[\int_{\Omega_{\mathbf{0}}\left(\mathcal{P}_{1}\right)} \log z d z\right] \\
\Longrightarrow \quad & \lim _{n \rightarrow \infty}\left[d \hat{S}_{\log }^{1}\left(X^{(n)} ; Y^{(M)}\right)+\log n\right]=-\int_{\mathbb{R}^{d}} f(z) \log g(z) d z+d \mathbb{E}\left[\int_{\Omega_{\mathbf{0}}\left(\mathcal{P}_{1}\right)} \log z d z\right], \\
& =H(f, g)+d \beta_{d, \log } . \\
& \beta_{d, \log } \doteq \mathbb{E}\left[\int_{\Omega_{\mathbf{0}}\left(\mathcal{P}_{1}\right)} \log z d z\right] .
\end{aligned}
$$

We hence see that in this case the unknown constant (which by analogy with the earlier case we will denote by $\beta_{d, \log }$ ), now appears as an additive rather than a multiplicative term. Also, we see that we obtain the cross-entropy $H(f, g)$ directly, rather than having to also take the $\lambda \rightarrow 0$ limit as in equation (11).

The constant $\beta_{d, \log }$ can be calculated using the approach detailed in the Appendix:

$$
\beta_{d, \log }=-\frac{1}{d}\left[\gamma+\log v_{d}\right], v_{d}=\frac{2 \pi^{\frac{d}{2}}}{d \Gamma\left(\frac{d}{2}\right)}, \gamma \doteq \lim _{n \rightarrow \infty}\left(\sum_{k=1}^{n} \frac{1}{k}-\log n\right)=0.577215664 \ldots,
$$

where $\gamma$ is the Euler-Mascheroni constant.

\section{Experiments}

We have the established the form of the specificity in the limit of infinite numbers of points. However, the question remains whether we can see the predicted behaviour for reasonable finite point numbers, both for $M$ and $n$.

\subsection{Scaling with $n$}

We first test the predicted scaling of the $\lambda$-specificity:

$$
\hat{S}_{\lambda}^{1}\left(X^{(n)} ; Y^{(M)}\right) \propto \frac{1}{n^{\frac{\lambda}{d}}} .
$$


The generating distributions $f(z)$ and $g(z)$ are taken to be simple rotationally symmetric gaußians in $\mathbb{R}^{d}$ centered at the origin, of varying variance. For the purposes of the experiment, at each instantiation we fix the sample set $Y$, and then also generate a large training set. Varying $n$ is then accomplished by taking just the first $n$ members of this large training set. Examples are shown in Figure 1, for std of $f(z)$ of 0.45 , and std of $g(z)$ of 0.5 . The sample set and large training sets were regenerated for each plot. It can be seen that $\log \hat{S}_{\lambda}^{1}$ does indeed scale linearly with $\log n$. Furthermore, the calculated slope of the best-fit straight line agrees with the predicted slope to within $9 \%$ for all the cases shown.

We also tested the predicted scaling behaviour of the $\log$-Specificity ${ }^{5}$ :

$\hat{S}_{\log }^{1}\left(X^{(n)} ; Y^{(M)}\right)=-\frac{1}{d} \log n+\frac{1}{d} H(f, g)+\beta_{d, \log }$.

Example plots of $\hat{S}_{\log }^{1}$ versus $\log n$ for various values of $d$ are shown in Figure 2, and in Figure 3, we compare the measured gradient over various instantiations of the large training set and sample set with the theoretical prediction above. As can be seen from the graphs, the $\log$-specificity does indeed vary with $n$ as predicted, even for these relatively small values of $n$, and that this behaviour persists across a

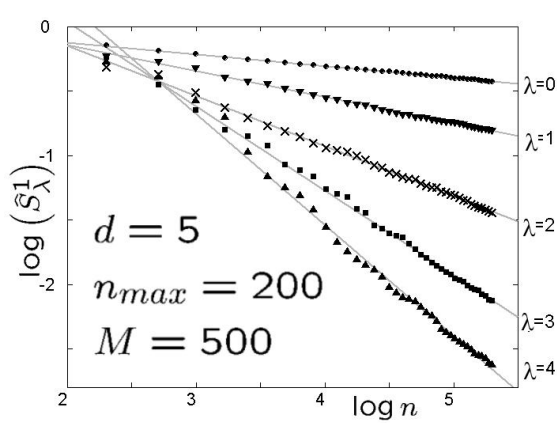

Figure 1: The $\log$ of the specificity $\hat{S}_{\lambda}^{1}$ plotted against $\log n$ for the case $d=5$, $M=500, n$ in the range 10 to 200 , for varying values of $\lambda$. range of values of $d$.

\subsection{Values of $\beta$}

As a check on our calculation, we consider simulations of unit intensity Poisson point processes in various numbers of dimensions. We have the prediction:

$$
\beta_{d, \log } \doteq \mathbb{E}\left[\int_{\Omega_{\mathbf{0}}\left(\mathcal{P}_{1}\right)} \log z d z\right]=-\frac{1}{d}\left[\gamma+\log v_{d}\right] .
$$

As noted earlier, in the simulations we can replace these integrals over Voronoi cells with the appropriate function of the nearest-neighbour distance. Results are shown in Figure 4. On the left, we show estimates of $\beta_{d, \log }$ calculated for $d=5$ for various numbers of simulation points $n$. For each point, we used 10 instantiations of the Poisson process. It would seem from this plot that the simulations converges very slowly. However, on the right we show the distribution of the log nearest-neighbour distances for a single instantiation. It can be seen that the difference between the predicted mean and the actual mean is actually reasonably small compared to the width of the distribution. We obtain similar results for other values of $d$.

\subsection{Comparison between Estimates and the Exact Results for $\tilde{D}_{\lambda}$.}

The limit given in eqn. (8) will not be valid for the case where the convex hull of the sample set $Y$ is substantially larger than the convex hull of $X^{(n)}$. This is because the points of $X^{(n)}$ lying on the periphery will have open Voronoi cells, which are populated by points of $Y$ (hence contribute to the specificity), yet whose size and shape is not as predicted by

\footnotetext{
${ }^{5}$ Care should be taken here to note the difference between the $\log$ of the $\lambda$-specificity $\left(\log \hat{S}_{\lambda}^{1}\right.$ ), and the $\log$-specificity $\left(\hat{S}_{\log }^{1}\right)$.
} 


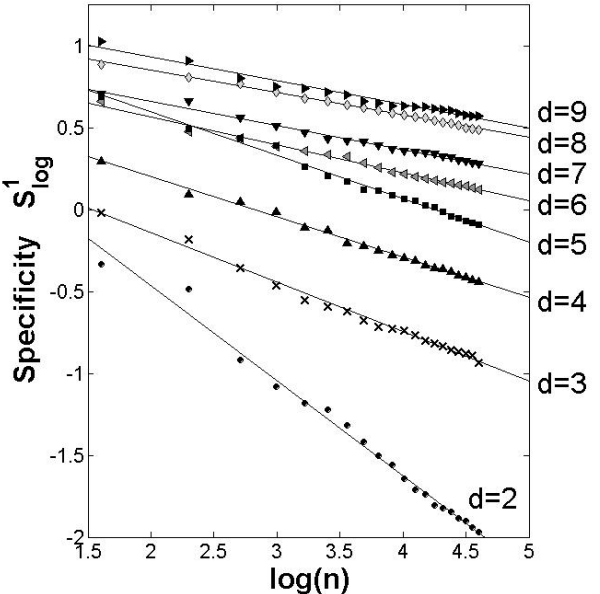

Figure 2: The log-specificity $\hat{S}_{\log }^{1}$ plotted against $\log n$ for the cases $d=2$ to $d=9$, a single instantiation of the large training set in each case. The sample set was of size $M=1000$, and the large training set of size $n$ in the range 10 to 100 . The lines are the straight-line fits to each graph.

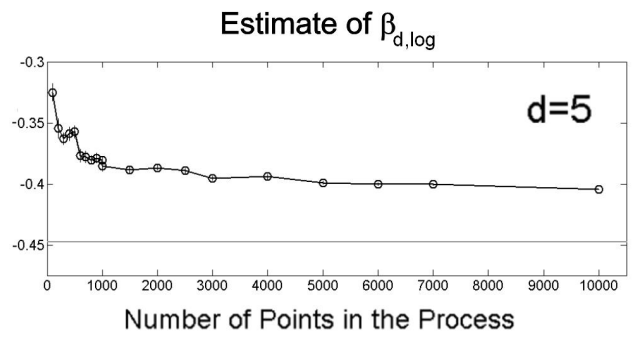

Figure 4: Left: $\beta_{d, \log }$ estimated from a uniform Poisson process in $d=5$ versus number of points in the simulation. The theoretical prediction is the horizontal gray line. Right: Distribution of log of the nearest-neighbour distances for single instantiations with $n=1000$ and $n=10000$ respectively (same horizontal scale for both). The actual mean is the grey line, the theoretical prediction the black line.

the above analysis. For the case where $f(z)$ and $g(z)$ are both gaußian distributions, the expression for $\tilde{D}_{\lambda}(f, g)$ (9) can be computed analytically. In the Figure, we show a comparison between our estimate of $\tilde{D}_{\lambda}(f, g)$ and the exact result. We used four datasets, each consisting of $n=100$ points drawn from a radially symmetric gaußian of width $\sigma_{g}=1$ in 5 dimensions.

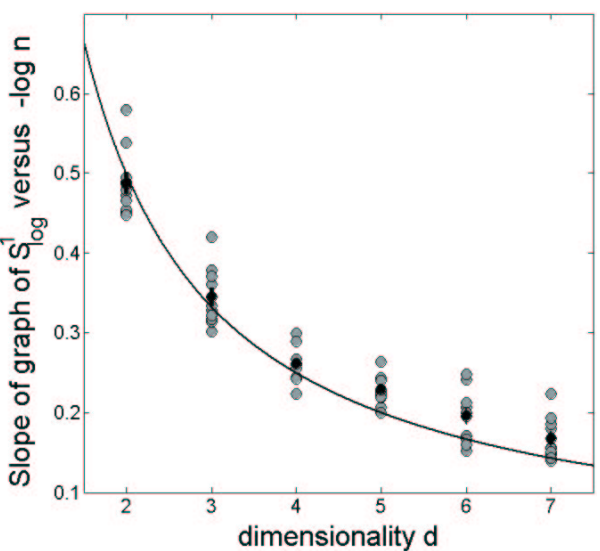

Figure 3: The slope of the graphs of logspecificity $\hat{S}_{\log }^{1}$ plotted against $-\log n$ as a function of the dimensionality $d$. The gray circles show the various instantiations, the black circles the mean values with standard error plotted. The line shows the theoretical prediction.

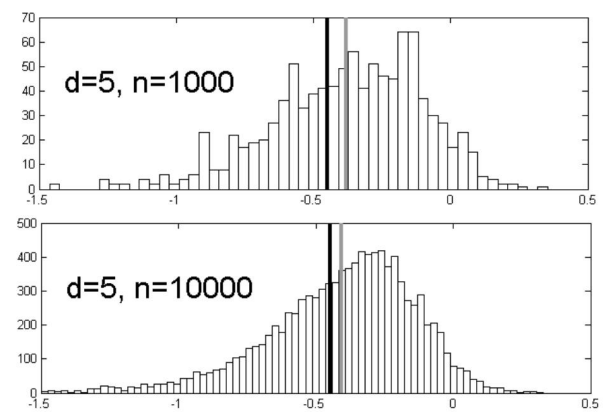


The divergence $\tilde{D}_{\lambda}(f, g)$ with $\lambda=0.1$ was then estimated ${ }^{6}$ from the Specificity for various values of the width of the model distribution $\sigma_{f}$. The sample sets $Y$ were of size $M=1000$, with the average value from 5 trials being taken for each point in the plot. It can be seen that as predicted, the estimated value of $\tilde{D}_{\lambda}(f, g)$ diverges from the exact theoretical prediction when the model is wider than the data (that is, for $\sigma_{f}>\sigma_{g}=1$ ). This Figure hence illustrates why we cannot use the estimated divergence $\tilde{D}_{\lambda}(f, g)$ to fit the model to the data. What we have done here is keep the data $g(z)$ fixed but varied the model $f(z)$. In practise, as noted earlier, we want to constrain the model (the model will always be fitted to the data), whilst varying the distribution of the data. That is, we wish to consider the effects of variations of the data at the point of fit. The agreement between the theoretical result and the estimate of $\tilde{D}_{\lambda}(f, g)$ at fit (the point $\sigma_{f}=1$ ) hence suggests that our analysis is valid at the required point.

\section{Discussion and Conclusions}

We have established the link between the generalised specificity and cross-entropy (hence also Kullback-Leibler divergence), and calculated the constants involved in closed form. We have tested the predicted scaling behaviour of the specificity, showing that this scaling holds good for even relatively small numbers of training points.

For the case of images, one pseudo-distance that has been previously used to define specificity is the shuffle distance. This makes specific use of the spatial properties of images. Let $I(p)$ be the value of image $I$ at pixel position $x$. Let $B(p, r)$ be the set of pixels contained in a ball of radius $r$ centered at $p$. The shuffle distance between two images $I$ and $J$ is then:

$$
\mathcal{D}_{r}(I, J) \doteq \sum_{p} \min _{q \varepsilon B(p, r)}|I(p)-J(q)| .
$$

This is not symmetric, but it is trivial to produce a symmetric version. Shuffle distance obviously scales linearly as we scale the image values, but it is obviously not translation invariant. Nor is it a monotonic function of the euclidean distance. Hence the above analysis cannot he applied to this instance. It may be that locally on the space of actual images, say, the shuffle distance may be approximately monotonic for small displacements, and approximately translation invariant for small displacements lying in the space of images (as opposed to displacements orthogonal to this). If so, then we would have an approximate relation of the same general form as that given for euclidean distances. However, this is at the moment just a conjecture, and is obviously highly data-dependant. Investigating this and other practical aspects of applying the estimators defined here is the focus of our current work.

\footnotetext{
${ }^{6} D_{\lambda}(f, g)$ as in eqn. (11), but without taking the limit $\lambda \rightarrow 0$, and $D_{\lambda}(f, f)$ from the exact result.
} 


\section{Appendix: Calculating the Expectation Value of Integrals over the Voronoi Cell.}

Consider equation (7) from the text:

$$
\beta_{d, \lambda} \doteq \mathbb{E}\left[\int_{\Omega_{\mathbf{0}}\left(\mathcal{P}_{1}\right)}(\|z\|)^{\lambda} d z\right]
$$

Rather than relating this integral to the expectation value for the powers of the nearestneighbour distance for uniform Poisson processes, we instead, as a check, perform the integral over the interior of the Voronoi cell explicitly. The naive way to calculate this expectation value would be to consider a given configuration of points of the process (seeds) about a seed point at the origin, evaluate this integral for the configuration of points, then perform the statistical average over the seed positions. However, following the methods of Brakke

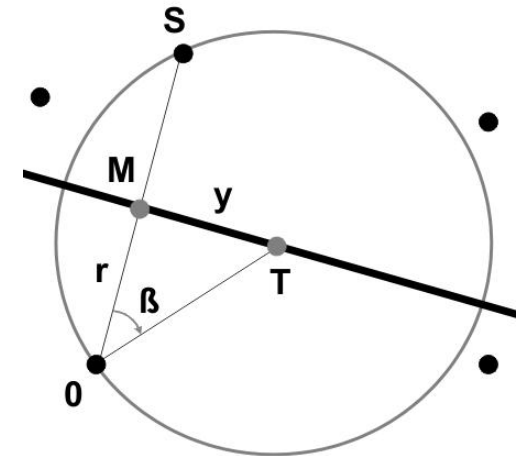

Figure 6: Black dots: Seed points, central seed 0 \& neighbouring seed S, Grey dots: The mid-point $\mathbf{M}$, possible face point $\mathbf{T}$, Thick black line: Possible face points on $(d-1)$-dimensional hyperplane, Grey line: Hypersphere through $\mathbf{0}$ and $\mathbf{S}$, centre $\mathbf{T}$. [1], it is simpler to combine these stages.

Consider the situation shown in Figure 6, for a fixed position of the seed $\mathbf{S}$. A point $\mathbf{T}$ lying on the plane midway between the seeds $\mathbf{0}$ and $\mathbf{S}$ is a point on a face of the Voronoi cell of $\mathbf{0}$ provided that no seed points lie in the hypersphere (gray line) centered on $\mathbf{T}$ that passes through the pair of seeds. If $\vec{y}$ is the position of $\mathbf{T}$ relative to $\mathbf{M}, y=|\vec{y}|$ and $r$ is the distance M0, then the void probability is just:

$$
\exp \left(-v_{d}\left(r^{2}+y^{2}\right)^{\frac{d}{2}}\right)
$$

where the bracketed term is the volume of the void. Let $d \vec{y}$ be the infinitesimal element of hyperarea $((d-1)$ dimensional volume lying wholly in the hyperplane) about $\mathbf{T}$. When joined to $\mathbf{0}$, this defines a hypercone, which forms part of the volume of the Voronoi cell. Since this cell is a convex polytope, integrating over all points $\mathbf{T}$ sweeps out the whole volume of the cell once and only once. We can hence calculate the contribution of this cone:

$$
\int_{0}^{\left(r^{2}+y^{2}\right)^{\frac{1}{2}}} \cos \beta \cdot R^{\lambda} \cdot\left(\frac{R}{\left(y^{2}+r^{2}\right)^{\frac{1}{2}}}\right)^{d-1} d R d \vec{y}=\frac{r}{d+\lambda}\left(r^{2}+y^{2}\right)^{\frac{\lambda}{2}} d \vec{y},
$$

where $\cos \beta=r /\left(r^{2}+y^{2}\right)^{\frac{1}{2}}$ projects $d \vec{y}$ perpendicular to $\mathbf{0 T}$, and $R$ takes us along the cone from $\mathbf{0}$ to $\mathbf{T}$. We then integrate over the angular part of $d \vec{y}$, which takes $\mathbf{T}$ over the surface of the $(d-1)$-dimensional hypersphere centered at $\mathbf{M}$ of radius $y$ to give:

$$
\frac{r}{d+\lambda} \cdot s_{d-1} y^{d-2} \cdot\left(r^{2}+y^{2}\right)^{\frac{\lambda}{2}} d y, \text { where: } s_{p} \doteq \frac{2 \pi^{\frac{p}{2}}}{\Gamma\left(\frac{p}{2}\right)},
$$

where $d y$ is now just the scalar measure. We now include the void probability and integrate over the position of the seed $\mathbf{S}$ :

$$
\int \frac{s_{d-1}}{d+\lambda} \cdot r y^{d-2} \cdot\left(r^{2}+y^{2}\right)^{\frac{\lambda}{2}} \exp \left(-v_{d}\left(r^{2}+y^{2}\right)^{\frac{d}{2}}\right) d y d \mathbf{S} .
$$


Note that the measure associated with the position of seed $\mathbf{S}$ is not a scalar. However, we can integrate over the angular part, taking $\mathbf{S}$ over the surface of a $d$-dimensional hypersphere of radius $2 r$ centered on $\mathbf{0}$ :

$$
\int_{\text {angular part }} d \mathbf{S}=s_{d} \cdot 2^{d} r^{d-1} d r
$$

The remaining variables to be integrated over are just the distances $y$ and $r$ :

$$
\beta_{d, \lambda}=\frac{s_{d} s_{d-1} 2^{d}}{d+\lambda} \int_{0}^{\infty} d r \int_{0}^{\infty} d y r^{d} y^{d-2} \cdot\left(r^{2}+y^{2}\right)^{\frac{\lambda}{2}} \exp \left(-v_{d}\left(r^{2}+y^{2}\right)^{\frac{d}{2}}\right) .
$$

Using the change of variables $r=l \cos \theta$ and $y=l \sin \theta$, this integral can be reduced to a standard form which can be evaluated, and after a little manipulation, gives the final result:

$$
\beta_{d, \lambda}=\frac{d}{(d+\lambda)} \cdot \Gamma\left(2+\frac{\lambda}{d}\right) \cdot\left(v_{d}\right)^{-\frac{\lambda}{d}} .
$$

As a final check on the normalisation, we note that $\beta_{d, 0}$ is just the expectation value of the volume of the Voronoi cell, which is correctly given as 1 by the above expression.

\section{References}

[1] Kenneth A. Brakke. Random voronoi tessellations in arbitrary dimension. http://www.susqu.edu/brakke/aux/downloads/papers/arbitrary.pdf, 2005 .

[2] R. H. Davies, C. J. Twining, P. D. Allen, T. F. Cootes, and C. J. Taylor. Shape discrimination in the hippocampus using an MDL model. In Proceedings of IPMI 2003, pages 38-50, 2003.

[3] A. Hero and O. Michel. Estimation of Rényi information divergence via pruned minimal spanning trees. IEEE Workshop on Higher Order Statistics, Caesaria, Israel, Jun. 1999.

[4] A.O. Hero, B. Ma, O. Michel, and J. Gorman. Applications of entropic spanning graphs. IEEE Signal Processing Magazine, 19(5):85-95, 2002.

[5] A.O. Hero, B. Ma, O. Michel, and J.D. Gorman. Alpha-divergence for classification, indexing and retrieval. Technical Rcport CSPL-328 Communications and Signal Processing Laboratory, The University of Michigan, 48109-2122, http://www.eecs.umich. edu/ ${ }^{\sim}$ hero/det_est.html, 2001.

[6] A. Rényi. On measures of entropy and information. Proceedings 4th Berkeley Symposium Math. Stat. and Prob., 1:547-561, 1961.

[7] J. M. Steele. Probability Theory and Combinatorial Optimization. SIAM, Philadelphia, 1997.

[8] Andrew R. Wade. Explicit laws of large numbers for random nearest-neighbour type graphs. http://arxiv.org/abs/math.PR/0603559, 2006. 